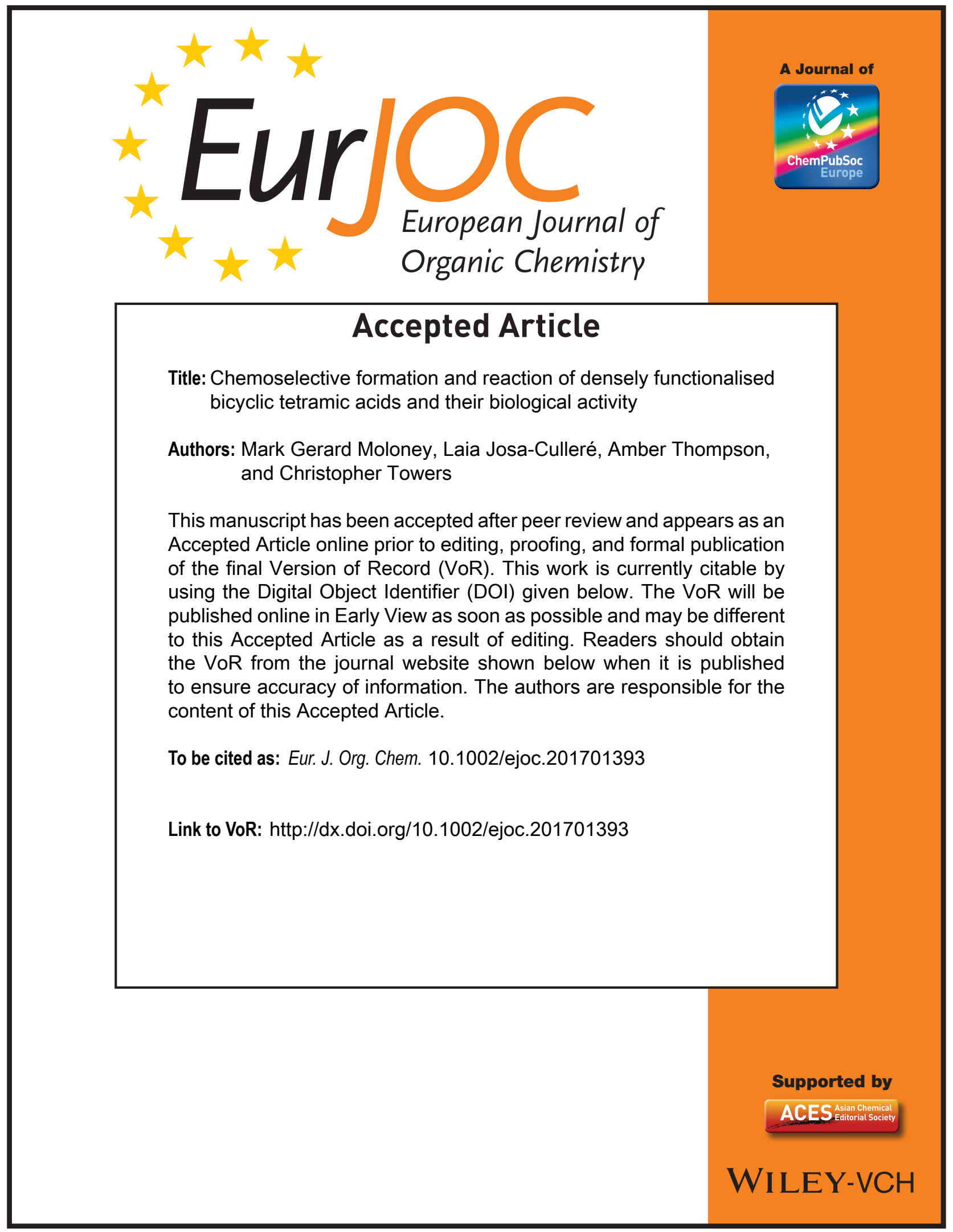




\title{
Chemoselective formation and reaction of densely functionalised bicyclic tetramic acids and their biological activity
}

\author{
Laia Josa-Culleré, ${ }^{[a]}$ Christopher Towers, ${ }^{[b]}$ Amber L. Thompson ${ }^{[a]}$ and Mark G. Moloney ${ }^{*[a]}$
}

\begin{abstract}
Weinreb amide derivatives of tetramates may be effectively accessed by chemoselective Dieckmann reaction, and further react with Grignards in a fully chemoselective reaction, giving rise to unsaturated acyl derivatives not easily available by other routes. These systems are strong chelators of calcium, and some show potent activity against Gram positive bacteria, and one is a first-in-class proteasome inhibitor.
\end{abstract}

The tetramic acid (pyrrolidine-2,4-dione) is the common structural unit in a class of natural products that possesses a wide range of biological activities including antibiotic, antifungal, antitumour and antiviral activity. ${ }^{[1]}$ Of particular interest are 3-acyl tetramic acids $\mathbf{1}$, found in natural products with potent antibacterial activities (Figure 1)..$^{[2]}$

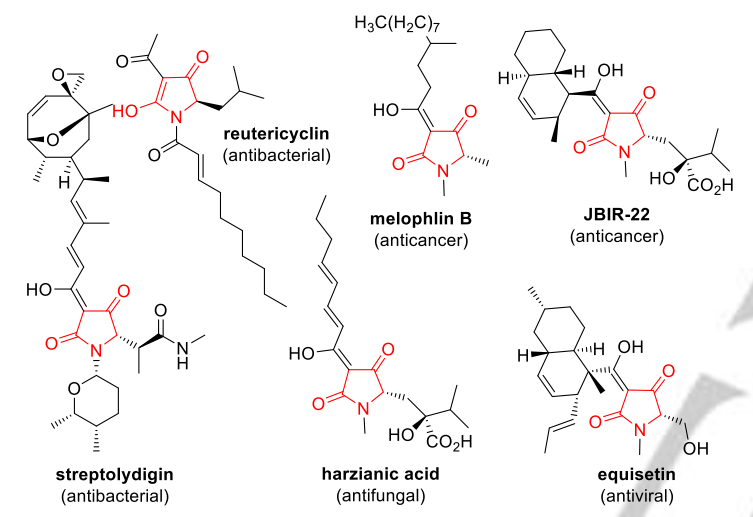

Figure 1. Representative examples of 3-acyltetramic acid-containing natural products.

Using the self-regeneration of stereocentre (SRS) principle, ${ }^{[3]}$ we have shown that rapid access the enantiopure tetramate cores 4a-c for three different systems, derived from serine ( $a: X=O$, $\mathrm{Y}=\mathrm{H}$ ), cysteine ( $\mathrm{b}: \mathrm{X}=\mathrm{S}, \mathrm{Y}=\mathrm{H}$ ) and threonine ( $\mathrm{c}: \mathrm{X}=\mathrm{O}, \mathrm{Y}=\mathrm{Me})$, is possible (Scheme 1). This synthesis relies on the Dieckmann cyclisation of $\mathrm{N}$-acylated oxazolidines 3a-c to give tetramates 4a-c as the major product. ${ }^{[4]}$ Although the tetramate core has no or little intrinsic biological activity, ${ }^{[5]}$ it can be functionalised to mimic features of tetramic acid-containing natural products, and some of these analogues have shown potent antibacterial activities against $E$. coli and $S$. aureus. ${ }^{[6]}$

[a] Dr L. Josa-Culleré, Dr A. Thompson, Prof M. G. Moloney Chemistry Research Laboratory

University of Oxford

12 Mansfield Road, OX1 3TA, Oxford (United Kingdom)

E-mail:mark.moloney@chem.ox.ac.uk

http://research.chem.ox.ac.uk/mark-moloney.aspx

[b] C. Towers

Department of Oncology

University of Oxford

Old Road Campus Research Building, OX3 7LJ, Oxford (United

Kingdom)

Supporting Information for this article is given via a link at the end of
We recently reported the preparation of acyltetramates from $4 a$ using the corresponding carboxylic acid and stoichiometric amounts of DCC-DMAP, which proceeded via O-acylation followed by rearrangement to $C$-acylated products $6 \mathbf{a}$, but which was found to be limited in substrate scope, and not suitable for vinylic substrates in particular. ${ }^{[7]}$ In order to enable the introduction of a wider range of functionalities to the tetramate core, we decided to prepare Weinreb amides 7a-c, for the introduction of the desired acyl group at $C(6)$ by Grignard reaction.
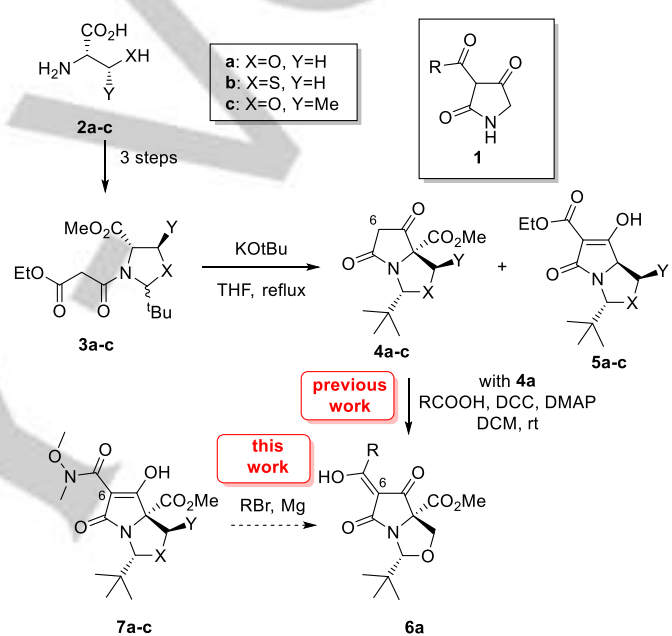

Scheme 1. Previous and novel approaches to acyl tetramic acids $\mathbf{6 a}$.

Our initial attempt (Approach 1, Scheme 2), was to directly acylate tetramate $\mathbf{4 a}$ using methoxy(methyl)carbamic chloride, ${ }^{[8]}$ but $\mathrm{O}$-acylated carbamate $\mathbf{8}$ was obtained as the only product, and attempted acyl migration with $\mathrm{DMAP}^{[0]}$ or acetone cyanohydrin $^{[10]}$ only delivered recovered tetramate $\mathbf{4 a}$, manifesting the good leaving group ability and low nucleophilicity of the tetramate system. In an alternative strategy (Approach 2, Scheme 2), acylation of oxazolidine $3 \mathbf{a}$ gave the desired tricarbonyl $9 a$, and this could be followed by Dieckman cyclisation under our previously reported conditions (with $\mathrm{KO}^{\mathrm{t}} \mathrm{Bu}$ ) or with piperidine to furnish a 1:1 mixture of Weinreb amide 7a and ethyl ester 10a (Table 1). However, DBU gave a better outcome, leading to exclusive formation of the desired tetramate $7 a$ in high yields and on a gram scale, and these conditions were also successful for $\mathbf{7 b}, \mathbf{c}$. This remarkable process provided access to the desired densely functionalised Weinreb derivatives 7a-b with near-complete chemoselectivity. 


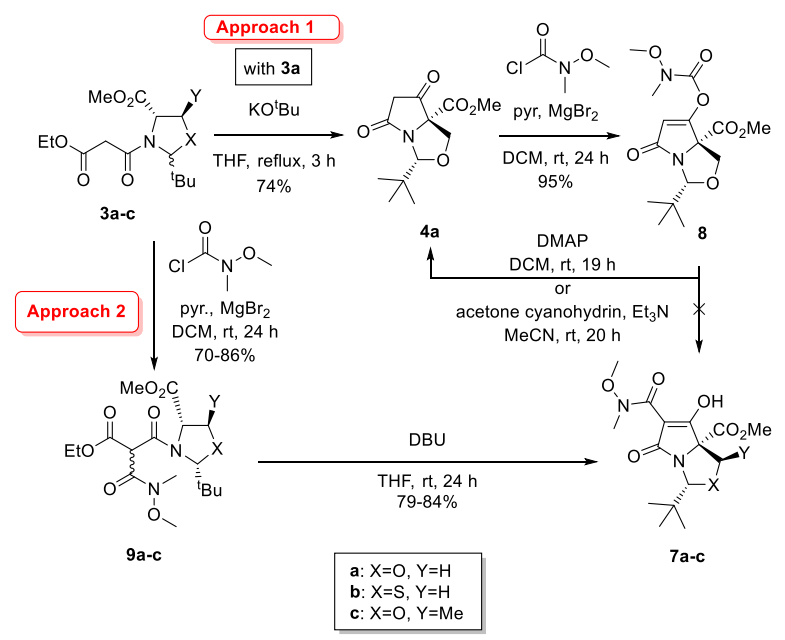

Scheme 2. Preparation of Weinreb amides 7a-c.

Table 1. Base screen for the cyclisation of tricarbonyl $9 a$ to $7 a / 10 a$.

\begin{tabular}{|c|c|c|c|c|c|}
\hline & $\mathbb{N} \quad \mathbb{N}^{\circ}$ & $\begin{array}{r}\text { base } \\
\text { THF, 5-2 }\end{array}$ & $=0$ & $\begin{array}{r}\text { Eto- } \\
+\quad 0\end{array}$ & $=\underbrace{\mathrm{OH}}_{. \mathrm{OH}}$ \\
\hline Entry & Base & $\mathrm{pK}_{\mathrm{a}}$ & Temp & Yield [\%] & Ratio 7a:10a \\
\hline 1 & Pyridine & 5.2 & $\mathrm{rt}$ & 0 & - \\
\hline 2 & $\mathrm{Et}_{3} \mathrm{~N}$ & 10.8 & $\mathrm{rt}$ & 0 & - \\
\hline 3 & Piperidine & 11.0 & $\mathrm{rt}$ & quant. & $1: 1^{[a]}$ \\
\hline 4 & DBU & 12 & $\mathrm{rt}$ & 84 & $110: 1^{[b]}$ \\
\hline 5 & $\mathrm{KO}^{\mathrm{t}} \mathrm{Bu}$ & 17 & Reflux & 76 & $1: 1^{[a]}$ \\
\hline
\end{tabular}

[a] Determined by NMR. [b] Determined by LC/MS

With Weinreb amides 7a-c in hand, treatment with 2 equivalents of the desired organomagnesium bromide gave acylated compounds $11-15 a-c$ in yields of up to $84 \%$ (Table 2), allowing the introduction of alkyl, aryl, alkenyl and alkynyl functionalities to the tetramate core. This remarkable reaction proceeds with full chemoselectivity on the extensively conjugated and crossconjugated substrates $7 \mathrm{a}-\mathrm{c}$, and with no reaction at the ketone or the methyl ester or over-addition products detected by NMR or MS analysis on the reaction product mixture.
Table 2. Grignard reaction of Weinreb amides 7a-c to give products 11-15.

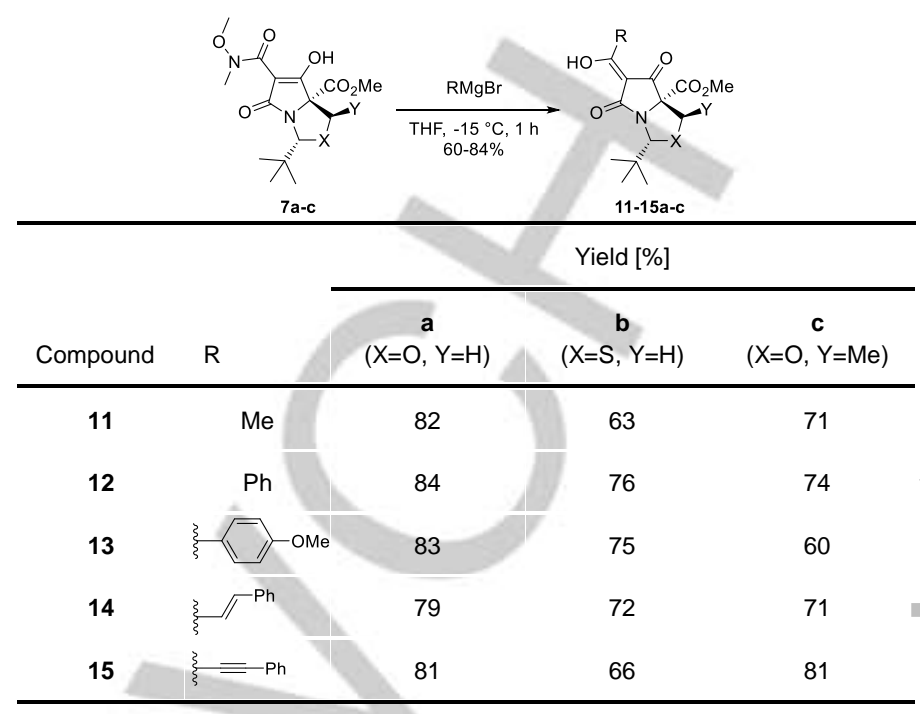

Weinreb amides 7a-c and the alkynyl analogues were found not to be fully stable, and after standing in $\mathrm{CDCl}_{3}$ for several days, enamine 16 and tricyclic compounds 17a-c were formed respectively (Scheme 3 ). The latter was confirmed from the crystal structure of $17 a^{[11]}$

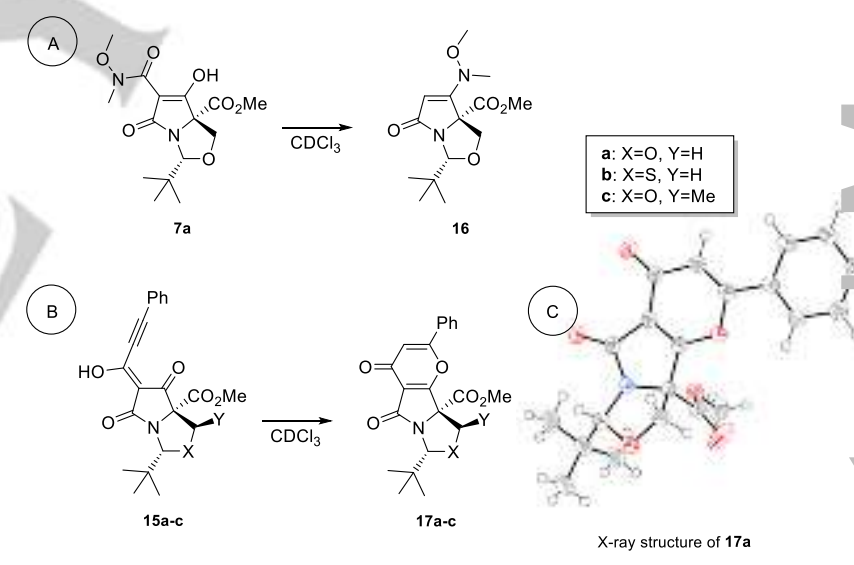

Scheme 3. Degradation products of $7 \mathrm{a}(\mathrm{A})$ and $15 \mathrm{a}-\mathrm{c}(\mathrm{B})$ in $\mathrm{CDCl}_{3}$ and crystal structure of tricyclic 17 a shown with displacement ellipsoids drawn at $50 \%$ probability; one molecule is shown for clarity (C).

${ }^{1} \mathrm{H}$ and ${ }^{13} \mathrm{C}$ NMR analysis of acylated compounds 11-15a-c in $\mathrm{CDCl}_{3}$ revealed that they exist as one major tautomer (along with a minor form observed in alkyl and alkenyl analogues 11a-c and 14a-c). The major form corresponds to the Z-exo-enol, while the minor one is the E-exo-enol (Figure S1); the ratio of each was solvent dependent (Figure S2). This assignment was confirmed from a ${ }^{1} \mathrm{H}-{ }^{13} \mathrm{C}$ NOE experiment of compound 11a in $\mathrm{C}_{6} \mathrm{D}_{6}$ (Supporting Information). In contrast, carboxamide- and alkoxy-carbonyl tetramic acids exist as the endo-enol form, reflecting their differing capacity for stabilising resonance. ${ }^{[12]}$ 


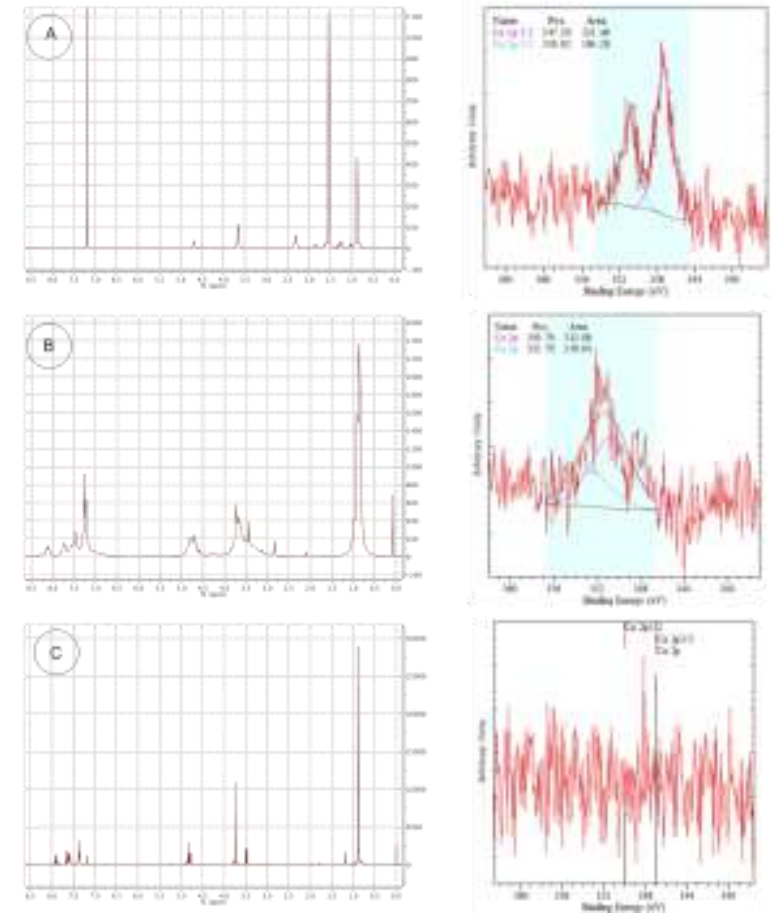

Figure 2. ${ }^{1} \mathrm{H}$ NMR $\left(400 \mathrm{MHz}, \mathrm{CDCl}_{3}\right.$ ) and XPS calcium region of 11a after silica purification (A), 14a after silica purification (B) and 14a after washing with $2 \mathrm{M} \mathrm{HCl}(\mathrm{C})$.

Purification of acyl-tetramic acids by column chromatography has been reported to be difficult, and this is believed to be due to the pronounced tendency of these compounds for chelation with metals. ${ }^{[1 c, 13]}$ In the work reported here-in, all acylated analogues gave broad NMR signals after silica gel purification, and needed to be washed with $2 \mathrm{M} \mathrm{HCl}$ to obtain metal-free samples with well-resolved NMR spectra (Figure 2). X-ray photoelectron spectroscopic (XPS) analysis of the chelated 11a and 14a clearly identified the presence of calcium in both cases, but no other metals (Mg, Fe, Co...) (Table S2). After washing 14a with $2 \mathrm{M} \mathrm{HCl}$ and analysing the solid metal-free sample again by XPS, no detectable amounts of calcium were found. Furthermore, when the metal-free sample, showing sharp NMR peaks, was washed with $2 \mathrm{M}$ aqueous $\mathrm{Ca}\left(\mathrm{NO}_{3}\right)_{2}$, broadening of the NMR signals was observed. These results indicate that the difficult purification of acyl tetramic acids by column chromatography may well be due to chelation of trace amounts of calcium found in the silica gel. In fact, some naturally occurring tetramic acids have been isolated as the metal complexes, and this chelation capacity could play a role in their potent biological properties. ${ }^{[14]}$

Removal of the tert-butyl group and the methyl ester protecting groups was of interest, not least because this would provide access to closer analogues of some natural products (Figure 1). However, N,O-acetal deprotection of acetylated compound 11a was unsuccessful. All the tested conditions, including treatment with 1,3-propanedithiol, ${ }^{[15]}$ used for the deprotection of similar compounds, ${ }^{[4 a, 16]}$ and other acidic conditions: $\mathrm{THF} / \mathrm{H}_{2} \mathrm{O}$ /formic acid $3: 1: 1,{ }^{[17]} \mathrm{TFA}(2 \mathrm{eq})$ in $\mathrm{THF} / \mathrm{H}_{2} \mathrm{O} 1: 1,{ }^{[18]} \mathrm{TFA}(20 \mathrm{eq})$ in DCM, and $\mathrm{BiBr}_{3}$ (cat.) in $\mathrm{MeCN}^{[19]}$ resulted in quantitative recovery of starting material. This lack of reactivity of the acylated products towards hydrolysis could be due to the presence of the exocyclic enol moiety, which stabilises the lactam by hydrogen bonding. ${ }^{[7]}$ This hypothesis was supported by the successful deprotection of Weinreb amide 7a (Scheme 4A), which has a free lactam carbonyl. To circumvent this problem, protection of the enol group was clearly necessary, but this also proved to be challenging due to the high stability - and low reactivity - of the derived enolate as a result of extensive resonance. Attempted silylation of 11a, or treatment with Mel in the presence of CsF, conditions reported for the successful $O$-alkylation of tetronic acids, ${ }^{[20]}$ gave unreacted starting material. Finally, treatment of 11a with methanol under Mitsunobu reaction conditions ${ }^{[21]}$ gave the methylated product, which was found to be a separable $3: 1$ mixture of enolates 19 and 19' (Scheme 4B), as indicated by HMBC correlations. For this protected enol, $\mathrm{N}, \mathrm{O}$-acetal deprotection was successful and alcohol 21 was obtained in $74 \%$ yield. However, hydrolysis of alkene 20 proved to be more challenging and purification by flash column chromatography was unsuccessful. This could be explained by a combination of factors, including its high polarity, metal chelation and a possible retro-aldol reaction. ${ }^{[22]}$ Indeed, purification of protected alcohol 23, prepared by direct acetylation of the crude 22, was successful.

Acylated analogues 11a and 14a-c were hydrolysed with an excess of $\mathrm{LiOH}$, and reflux of the crude acids in $\mathrm{MeCN}$ gave the decarboxylated products $\mathbf{2 4}$ and $\mathbf{2 5 a - c}$ as single diastereomers. The exo-located tert-butyl group probably plays an important role in the selectivity observed in these processes.

The biological activity of acylated compounds 11a-c - 14a-c and Weinreb amides 7a-c against Gram-positive Staphylococcus aureus and Gram-negative Escherichia coli was initially assessed using the hole-plate method, which allows a direct qualitative assessment of antibacterial activity (Table S3). ${ }^{[2 b]} \mathrm{We}$ found a high dependence of activity on the nature of the acyl substituent, with Weinreb amides, alkyl and aryl groups showing no or little inhibition; however, high $S$. aureus activity of the alkenyl analogues 14a-c, and particularly $14 \mathrm{~b}$ was found. Similar selectivity for Gram-positive bacteria had been previously observed for related substituted tetramates. ${ }^{[6-7,23]}$ For 17a-c, some inhibition of both $S$. aureus and Gram-negative E. coli was observed. However, deprotected compounds 21 and 23 were fully inactive. Conversely, decarboxylation led to an improvement of Gram-positive activity: acetyl $\mathbf{2 4}$ showed some activity at $4 \mathrm{mg} / \mathrm{mL}$, while its analogous methoxycarbonyl 11a had been inactive, and the alkenyl analogues also displayed enhanced activities. The cysteine analogues were again superior, and the most active analogue $25 \mathrm{~b}$ was 27 -fold more potent than the standard CephC. MIC analysis (Table S4) confirmed this analysis, with good activity against Gram-positive $S$. aureus and $S$. pneumoniae, but no Gram-negative activity. The most active analogue $25 \mathrm{~b}$ has MIC of $0.98 \mu \mathrm{g} / \mathrm{mL}$. These results validate the effectiveness of the hole-plate method, which despite not providing accurate MIC values, represents a valuable rapid initial assay to rank analogue activity. 


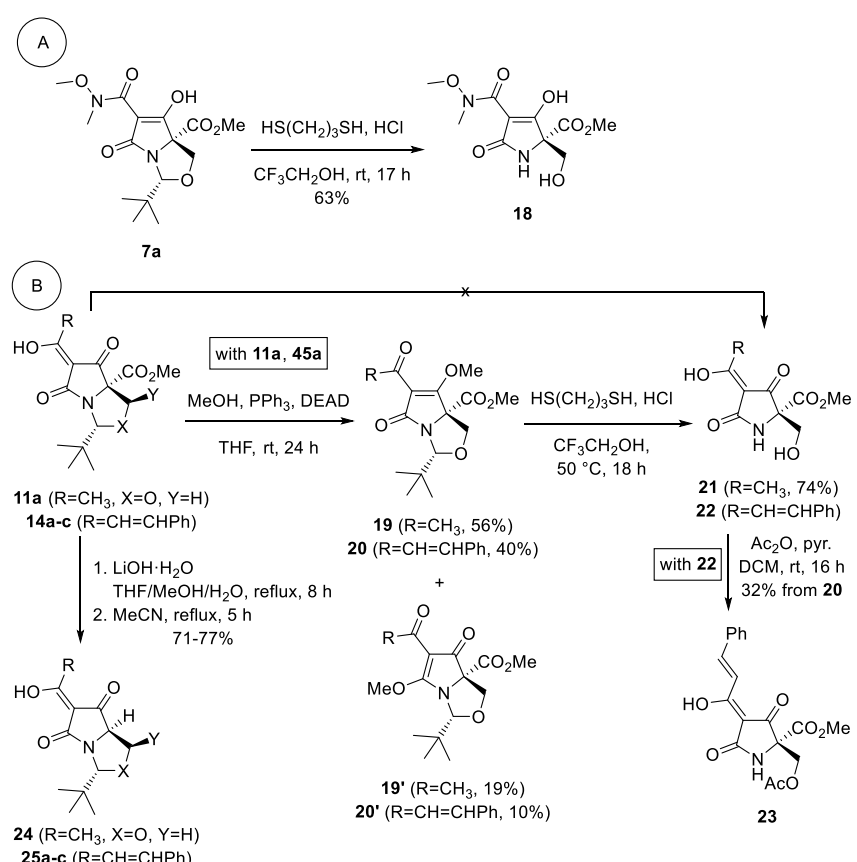

Scheme 4. N,O-Acetal deprotection and hydrolysis of 11a and 14a-c.

SAR analysis using predicted physicochemical properties was conducted (Table S5). The most active compounds are the ones with the highest clogP values and are clustered in a very tight zone of chemical space (Figure 3), a phenomenon which has been observed in other related systems. ${ }^{[6]}$ A previous study on related $C(6)$-acyltetramates ${ }^{[6]}$ found that those with $\operatorname{clog} \mathrm{P}>3$ and MSA > 620 tended to be more easily transported by the efflux pump in Gram-negative $H$. influenzae. That the most active tetramates synthesised herein with selective Grampositive activity behaved similarly and have clogP $>3$ and MSA $>450$ suggests that the observed lack of activity of these systems against $E$. coli could be ascribed to efflux pump (and/or lack of cell permeability) rather than a lack of intrinsic bioactivity.

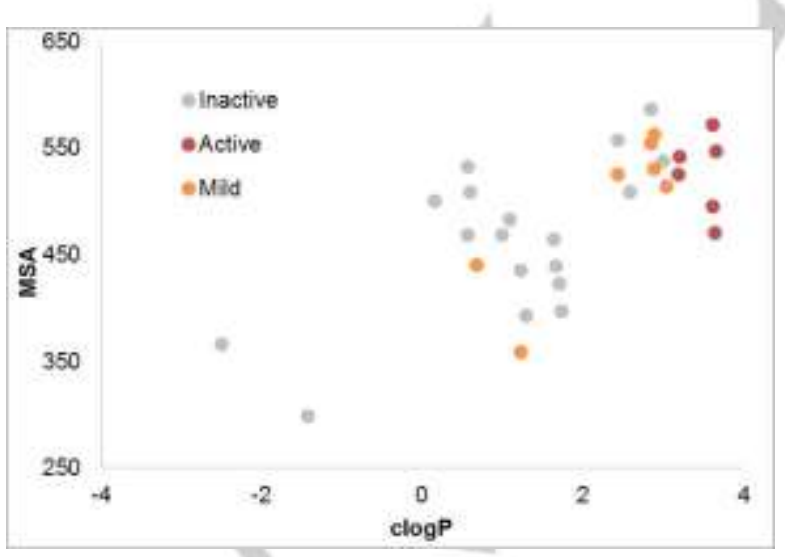

Figure 3. Correlation between the Gram-positive activity of the synthesised analogues and their MSA and clogP values.
The synthesised analogues were also studied for anticancer activity; an initial high-throughput screen gave alkenyl analogues 25a and $\mathbf{2 5 b}$ as the only hits, of interest since these were also the ones with the highest antibacterial activity. $\mathrm{IC}_{50}$ values against the five cancer cell lines (HeLa, H460, MCF7, SKOV-3 and $\mathrm{KMS}-12 \mathrm{BM}$ ) were found to be in the 10-20 $\mu \mathrm{M}$ range (Table S6). The mechanism of action of these compounds was investigated for apoptosis, caspase activation and proteasome inhibition. The most active compound, $25 \mathrm{~b}$, was found to be a reversible proteasome inhibitor $\left(\mathrm{IC}_{50} 25 \mu \mathrm{M}\right)$ in $\mathrm{H} 460$ cells (Table S7, Figure S3). The natural tetramic acid JBIR-22 has also been shown to inhibit the PPI of PAC3 homodimer, which plays a role in proteasome formation, and it could be that $25 \mathrm{~b}$ is acting via the same mechanism. Although JBIR-22 was also shown to be cytotoxic to HeLa cells with $\mathrm{IC}_{50} 68 \mu \mathrm{M},{ }^{[2]]}$ its proteasome activity has not been assessed. This work represents the first reported evidence of a tetramic acid acting as a proteasome inhibitor.

In conclusion, a fully chemoselective Grignard displacement of Weinreb amides $7 a-c$ has enabled the preparation of tetramic acids substituted with a range of side chain acyl groups; this alternative strategy to the classical acylation approach ${ }^{[7,12]}$ offers a solution to the recently highlighted difficulty of the introduction of unsaturated side chains in such systems, ${ }^{[24]}$ and neatly complements the work of Schobert which has demonstrated the suitability of Lacey-Dieckman cyclisation in highly sensitive substrates. ${ }^{[2]}$ Some of these analogues had potent and selective activity against Gram-positive bacteria, and one is a first-in-class proteasome inhibitor. This outcome supports the hypothesis that preparation of simple, and more easily accessible, mimics of tetramate-containing natural products can lead to active compounds suitable for drug discovery and development.

\section{Acknowledgements}

We gratefully thank SeaLife Pharma (Austria) for MIC determinations. LJC received funding from the People Programme (Marie Curie Actions) of the European Union's Seventh Framework Programme (FP7/2007-2013) under REA grant agreement no 316955. CT was supported by funding to the Preclinical Validation Lab from the Cancer Research UK Oxford Cancer Research Centre. This work was supported by Cancer Research UK (CRUK) grant number C38302/A13012, through the Cancer Research UK Oxford Centre.

Keywords: tetramate $\cdot$ Weinreb $\cdot$ chemoselective $\cdot$ antibacterial - antiproteasomal

[1] aB. J. L. Royles, Chem Rev 1995, 95, 1981-2001; bR. Schobert, A Schlenk, Bioorg Med Chem 2008, 16, 4203-4221; cM. Petermichl, R. Schobert, Synlett 2017, 28, 654-663.

[2] aS. Tuske, S. G. Sarafianos, X. Wang, B. Hudson, E. Sineva, J. Mukhopadhyay, J. J. Birktoft, O. Leroy, S. Ismail, A. D. Clark, Jr., C. Dharia, A. Napoli, O. Laptenko, J. Lee, S. Borukhov, R. H. Ebright, E. Arnold, Cell 2005, 122, 541-552; bM. G. Ganzle, Appl Microbiol Biotechnol 2004, 64, 326-332; cS. B. Singh, D. L. Zink, M. A. Goetz, A. W. Dombrowski, J. D. Polishook, D. J. Hazuda, Tetrahedron Lett 1998, 39, 2243-2246; dF. Vinale, G. Flematti, K. Sivasithamparam, M. Lorito, R. Marra, B. W. Skelton, E. L. 
Ghisalberti, J Nat Prod 2009, 72, 2032-2035; eS. Aoki, K. Higuchi, Y. Ye, R. Satari, M. Kobayashi, Tetrahedron 2000, 56, 1833-1836; fM. Izumikawa, J. Hashimoto, T. Hirokawa, S. Sugimoto, T. Kato, M. Takagi, K. Shin-Ya, J Nat Prod 2010, 73, 628-631.

[3] D. Seebach, A. R. Sting, M. Hoffmann, Angew Chem Int Edit 1996 35, 2708-2748.

[4] aM. D. Andrews, A. G. Brewster, K. M. Crapnell, A. J. Ibbett, T. Jones, M. G. Moloney, K. Prout, D. Watkin, J Chem Soc Perk T 1 1998, 223-235; bY. C. Jeong, M. Anwar, T. M. Nguyen, B. S. W. Tan, C. L. L. Chai, M. G. Moloney, Org Biomol Chem 2011, 9 , 6663-6669.

[5] Y. C. Jeong, M. G. Moloney, Synlett 2009, 2487-2491.

[6] Y. C. Jeong, M. Anwar, Z. Bikadi, E. Hazai, M. G. Moloney, Chem Sci 2013, 4, 1008-1015.

[7] S. W. B. Tan, C. L. L. Chai, M. G. Moloney, Org Biomol Chem 2014, 12, 1711-1716.

[8] D. Sucunza, D. Dembkowski, S. Neufeind, J. Velder, J. Lex, H. G. Schmalz, Synlett 2007, 2569-2573

[9] Y. C. Jeong, M. G. Moloney, J Org Chem 2011, 76, 1342-1354.

[10] M. Abe, T. Imai, N. Ishii, M. Usui, Biosci Biotechnol Biochem 2006 70, 303-306.

[11] Single crystal diffraction data were collected using a (Rigaku) Oxford Diffraciton SuperNovaA Diffractometer. Raw frame data were collected and reduced using CrysAlisPro; the structure was solved using SuperFlip [J. Appl. Cryst. 2007, 40, 786-790] and refined using CRYSTALS [J. Appl. Cryst. 2003, 36, 1487; J. Appl. Cryst. 2010, 43, 1100-1107]. See the SI (CIF) for further information. Crystallographic data have been deposited with the Cambridge Crystallographic Data Centre as supplementary publication number CCDC 1578211.

[12] Y. C. Jeong, M. G. Moloney, Beilstein J Org Chem 2013, 9, 18991906.

[13] aM. Sodeoka, R. Sampe, S. Kojima, Y. Baba, N. Morisaki, Y Hashimoto, Chem Pharm Bull (Tokyo) 2001, 49, 206-212; bB.
Barnickel, R. Schobert, J Org Chem 2010, 75, 6716-6719; cR. Schobert, Naturwissenschaften 2007, 94, 1-11.

aM. H. Lebrun, P. Duvert, F. Gaudemer, A. Gaudemer, C.

Deballon, P. Boucly, J Inorg Biochem 1985, 24, 167-181; bE. TeliKokalari, V. Stefanou, D. Matiadis, G. Athanasellis, O. Igglessi-

Markopoulou, S. Hamilakis, J. Markopoulos, Fresen Environ Bull

2012, 21, 3215-3223.

E. J. Corey, G. A. Reichard, J Am Chem Soc 1992, 114, 1067710678.

[16] aP. Angelov, K. M. Hosamani, Y. C. Jeong, M. G. Moloney, A. L. Thompson, M. Yaqoob, Synlett 2011, 2181-2184; bM. Anwar, M. G. Moloney, Tetrahedron Lett 2007, 48, 7259-7262; cC. A. Holloway, C. J. Matthews, Y. C. Jeong, M. G. Moloney, C. F. Roberts, M. Yaqoob, Chem Biol Drug Des 2011, 78, 229-235.

J. S. Panek, C. E. Masse, J Org Chem 1998, 63, 5294-5294.

N. Langlois, A. Moro, Eur J Org Chem 1999, 3483-3488.

X. Cong, F. Hu, K. G. Liu, Q. J. Liao, Z. J. Yao, J Org Chem 2005, 70, 4514-4516.

T. Sato, K. Yoshimatsu, J. Otera, Synlett 1995, 843-844

J. S. Bajwa, R. C. Anderson, Tetrahedron Lett 1990, 31, 6973 6976.

[22] M. G. Moloney, M. Yaqoob, Tetrahedron Lett 2008, 49, 6202-6204. [23] R. Yendapally, J. G. Hurdle, E. I. Carson, R. B. Lee, R. E. Lee, J Med Chem 2008, 51, 1487-1491.

[24] J. D. Myrtle, A. M. Beekman, R. A. Barrow, Org Biomol Chem 2016 14, 8253-8260.

[24] M. Petermichl, S. Loscher, R. Schobert, Angew Chem Int Edit 2016, 55, 10122-10125. 
Entry for the Table of Contents (Please choose one layout)

Layout 1:

\section{COMMUNICATION}

Text for Table of Contents

Layout 2:

\section{COMMUNICATION}

Author(s), Corresponding Author(s)*

\section{Page No. - Page No.}

Title

((Insert TOC Graphic here))

\section{((Insert TOC Graphic here))}

Author(s), Corresponding Author(s)* Page No. - Page No.

Title 